\title{
La corrupción a gran escala no tipificada en el ordenamiento colombiano: análisis desde los escenarios jurídicos y categorías de corrupción
}

\author{
Recibido: 23 de julio de 2020 - Aprobado: 8 de septiembre de 2020 \\ https://doi.org/10.22395/ojum.v20n42a5
}

Leónidas Pino Cañaveral

Fundación Universidad Católica Lumen Gentium, Cali, Colombia tertulia.cali07@gmail.com https://orcid.org/0000-0002-1855-2036

Alex Garcés Medrano

Fundación Universidad Católica Lumen Gentium, Cali, Colombia garcesmedrano@gmail.com https://orcid.org/0000-0002-8537-9448

Tirson Mauricio Duarte Molina

Diacronía: Centro de Estudios Sociales y Humanísticos, Cali, Colombia tirsonmduarte@gmail.com https://orcid.org/0000-0002-6240-2609

Juan David Mambuscay Burbano

Diacronía: Centro de Estudios Sociales y Humanísticos, Cali, Colombia mambuscay.jd@gmail.com https://orcid.org/0000-0003-1866-7244

\section{RESUMEN}

El presente artículo tiene por objetivo describir la corrupción como un fenómeno social que surge entre las relaciones del Estado como regulador y los particulares como destinatarios a partir de la imbricación de diferentes acciones, particularmente de la legitimidad institucional en las esferas pública y privada. Metodológicamente se toma como referencia la tipificación penal para efectos de establecer los escenarios posibles (públicos y privados) donde se ejecutan acciones de corrupción en varios niveles. Se analiza cada delito conforme a una escala mínima, media o amplia que sirva de insumo para comprender la dinámica de la corrupción y su relación con el Estado. A este fin se vincula una noción propia de corrupción a gran escala, instante poco analizado y de suma importancia para el ordenamiento jurídico colombiano. Se encuentra, entonces, que las situaciones materiales no encuentran correspondencia punitiva con la norma existente o desbordan sus límites entre los delitos tipificados. Por ello, instan por su inclusión como modalidad específica.

Palabras clave: Estado; corrupción; delitos; administración pública; corrupción a gran escala; tipo penal; escenarios sociales. 


\title{
Not-typified Large-Scale Corruption in the Colombian Ordering: an Analysis from the Judicial Scenarios and Corruption Categories Abstract
}

\begin{abstract}
The objective of this article is to describe corruption as a social phenomenon that arises between the relations of the State as regulator and individuals as recipients, from the interweaving of different actions, particularly of institutional legitimacy in the public and private spheres. Methodologically, the criminal classification is taken as a reference in order to establish the possible scenarios (public and private) where corruption actions are carried out at various levels. Each crime is analyzed according to a minimum, medium or broad scale, trying to serve as an input to understand the dynamics of corruption and its relationship with the State. To this end, a notion of Large-Scale Corruption is linked, an instant little analyzed and of great importance for the Colombian legal system. It is then found that the material situations do not find punitive correspondence with the existing norm or exceed their limits among the typified crimes, thus urging their inclusion as a specific modality.
\end{abstract}

Keywords: State; corruption; crimes; public administration; large-scale corruption; criminal type; social scenarios. 


\section{INTRODUCCIÓN}

Este artículo de investigación tiene como marco principal lo trabajado en el proyecto La legitimidad institucional en Colombia: análisis al involucramiento del poder judicial en fenómenos de corrupción a gran escala del Grupo de Investigación en Derecho y Ciencias Políticas de la Fundación Universidad Católica Lumen Gentium. De este proyecto se obtuvieron como resultado las siguientes inferencias concernientes al tema presentado como piedra angular de análisis.

Pensar en lo público como esencia definitoria del Estado y sus instituciones ocasiona que, tanto su actuación como sus omisiones, repercutan en el acuerdo común sobre el cual está cimentado desde un sentido hobbesiano de la situación. Al mismo tiempo, la otra parte, los signatarios — ciudadanos - también se encuentran sometidos a una regulación de su conducta por medio de leyes, normas u otras disposiciones que establecen límites a todo acto que atente con desestabilizar la armonía social. Esta relación bidireccional encuentra fundamento en la legitimidad que los unos otorguen a los otros, toda vez que éstos últimos satisfagan las necesidades por las que fueron creados - o aceptados - en una suerte de ejercicio virtuoso y ejemplarizante. Teóricamente, suena inefable.

En la realidad, el caso latinoamericano apunta a ser más difuso e intrincado. Ya señalan Seligson y Smith (2010) que el grueso poblacional de países del hemisferio desconfía de las instituciones que son clave en los regímenes democráticos, tales como los partidos políticos o las corporaciones legislativas. Los primeros son puente esencial en la representatividad del pueblo y sus ideas, mientras que las segundas son el espacio donde se elevan como leyes lo considerado común a todos. Entre tantos motivos sobre los que reposa este malestar, el fenómeno de la corrupción actualmente es transversal a todos ellos. No en vano se afirma que los gobiernos de América Latina, a pesar de elaborar estrategias y llevar esfuerzos magnos por contrarrestarlo, siguen fallando en la misión, lo que perpetúa una sensación de desgobierno y fragilidad (Isaza, 2012). Al buscar una mayor especificidad de los casos, el contexto colombiano no dista en señalar mejora: conforme a los resultados manifiestos en Gallup Colombia (2019), un $74 \%$ de la población encuestada señala la corrupción como un problema en aumento.

Sobre este fenómeno en el país, ya asegura Ospina (2013) que su inicio ocurrió en el gobierno de Alfonso López Michelsen (1974-1978). En palabras del escritor colombiano, afirma: "En cambio, algunos males nuevos se añadieron durante su gobierno a los males que ya nos había dejado el Frente Nacional: allí nació la corrupción [...] a partir de López, la corrupción empezó a hacer carrera" (Ospina, 2013, pp. 199-200). Bajo esta idea y lo que la actualidad nos expresa, la presente investigación estará orientada a escudriñar en el fenómeno de la corrupción en Colombia y sus nuevas características en el sector público, toda vez que las altas esferas e instancias del poder estatal se han visto involucradas. Con ello, ha distorsionado la forma de percibirlo desde el derecho, 
lo que hace urgente una nueva tipificación legal que las aborde. En las palabras de Carnelutti (2013): "La evolución del ordenamiento jurídico es, precisamente, en el sentido del empleo de la pena a los fines de reprimir una variedad cada vez mayor de las llamadas conductas antisociales" (p. 28). Del mismo modo, tal evolución acoge al derecho penal dado que, como se ha previsto, la crisis del sistema deviene de la incapacidad en las doctrinas existentes por dar solución a los nuevos conflictos y tendencias delictivas. Por ello, instan a un replanteamiento de las perspectivas con que son abordadas (Vallejo, 2011).

En este sentido, la primera parte de este escrito estará enfocada en explicar la metodología de construcción de escenarios, toda vez que su uso será expuesto como parte central de esta investigación. Para alimentar las variables que la metodología planteada requiere, un segundo ejercicio consta en definir qué se entiende por corrupción, en el que se aprovechan las múltiples acepciones del término construidas por teóricos, organizaciones internacionales y nacionales, entre otros actores. En este mismo aparte, se hará una recopilación de los elementos teóricos que puedan obtenerse alrededor del concepto de corrupción a gran escala. Esta recopilación nos permite asegurar que en este concepto se recogen las características que toma el fenómeno de la corrupción en las altas esferas del poder colombianas, lo que genera nuevos retos y riesgos para la consolidación de la democracia y su ordenamiento jurídico. Por último, se aplicará la metodología postulada para buscar los escenarios en donde los delitos tipificados en Colombia, como sinónimos de corrupción, puedan o no tener estrechez con el fenómeno de la corrupción a gran escala.

\section{METODOLOGÍA}

Ante la necesidad de establecer un concepto propio a la tipología de corrupción que integre agentes públicos y agentes privados, tanto a nivel nacional como internacional, se usará la metodología de construcción de escenarios para determinar la compatibilidad del tipo penal atribuido a corrupción privada, así como a los casos diversos y aún no caracterizados que se han tratado como comportamientos aislados constitutivos de falta disciplinaria. Si nos adentramos en materia penal, se mantiene este vacío en el entendido de que no existe un título o capítulo en el Código Penal que refiera a delitos propios de corrupción.

A partir de este método se puede analizar el entorno y comparar diferentes factores. Se trata de una técnica para establecer posibilidades y determinar decisiones. La construcción de escenarios tiene en cuenta cada posibilidad y el desarrollo futuro de cada caso, con el cual se logra un apoyo metodológico y epistémico (Londoño y Marín, 2002). A partir de los escenarios, el fenómeno en estudio se analiza desde un punto de vista retrospectivo y actual (Cely, 1999). Se usarán como variables el concepto de corrupción mismo, el tipo penal correspondiente a corrupción privada y algunos tipos que se pueden atribuir a los agentes públicos. Asimismo, se utilizarán 
las nociones que se han establecido alrededor del concepto de corrupción y sus diferentes acepciones: micro, meso o macro.

Desde una óptica tradicionalista, se debe comprender como escenario todo lo que está al alcance de la imaginación. Sin embargo, este concepto tiene dos vertientes: I) los escenarios realizables donde se estructura todo aquello que es plausible en la medida en que las condiciones reales de restricción sean eliminadas, y II) los escenarios deseables que hacen parte del concepto general, pero que las condiciones no son propicias para que sea realizable (Maqueda, 1996; Rúa, Duarte y Gómez, 2019). De acuerdo con ello, Rúa Duarte y Gómez (2019) afirman:

Como extensión de lo anterior, se encuentran que [...] los deseables, a los cuales los sujetos desean llegar, también pueden ser calificados como los escenarios más convenientes en relación con las consecuencias; y los realizables son aquellos en los que la ocurrencia es factible en relación con las limitaciones de todas las demás posibilidades, es decir, todos los factores llevan a este futuro sin que necesariamente sea conveniente para los involucrados (Cely, 1999). Se advierte que los escenarios deseables forman parte de los escenarios posibles y no necesariamente son realizables. (p. 121)

En suma, la construcción de escenarios sirve para profundizar el conocimiento sobre el presente y sus tendencias a partir de supuestos teóricos (Gutman, 1995). Estas suposiciones pueden organizarse en un modelo nuevo. Los escenarios apuntan a establecer y controlar relaciones de incertidumbre para sugerir ajustes y medidas de contingencia (Gutman, 1995). Crear escenarios no puede ser un ejercicio aislado.

Consecuentemente, y teniendo como fin de la construcción de los escenarios, se seleccionaron los tipos penales correspondientes a los delitos contra la Administración pública (P) en cada una de sus modalidades: peculado (P1), concusión (P2), cohecho (P3), celebración indebida de contratos (P4), tráfico de influencias (P5), enriquecimiento ilícito (P6) y prevaricato (P7). No obstante, por motivos de economía del espacio, únicamente se tratará P1, P2, P5 y P6, sin llegar a obviar la importancia de las demás conductas en la construcción teórica, postergándolas así a un segundo momento de revisión. Se aplicarán a estas variables en correspondencia con los diferentes niveles de corrupción: micro (M1), meso (M2) y macro (M3). De acuerdo con lo anterior, y atendiendo lo planteado por Vargas (2017) y Rúa, Duarte y Gómez (2019), las variables se asignan de acuerdo con el método de cadena del razonamiento inductivo como una forma de construcción de argumentos que a su vez sirven como parámetros del escenario mismo. Esto se debe a que se logra la construcción de un argumento mediante un hecho y se abarcan las posibilidades que se pueden alcanzar, de allí la utilidad de la metodología de los escenarios, pues "estas probabilidades inductivas sí pueden ser comparadas. [...]. Esto permite pasar de la veracidad a la objetividad" (Vargas, 2017, pp. 1028-1029). La distribución se dará de la siguiente manera (figura 1): 


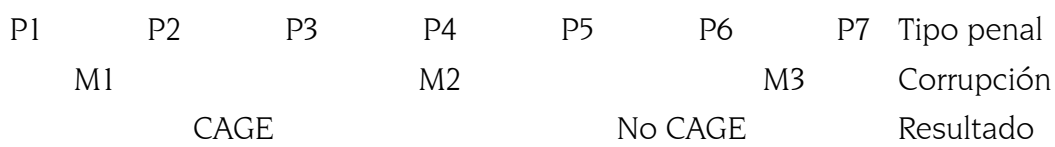

Figura 1. Esquema para la construcción de escenarios

Fuente: elaboración propia a partir de Rúa, Duarte y Gómez (2019).

Como resultado, se obtendrá el escenario realizable sobre la adecuación o no de estos tipos a las teorías sobre corrupción establecidas. Desde ello, se ostenta por ampliar el espectro a partir del cual es entendido el fenómeno a través de sus distintas modalidades y sujetos involucrados y, conforme a esto, se establecen los cimientos necesarios para su tipificación a nivel penal. De esta manera, se busca una mayor incidencia sobre el delito en cuestiones de judicialización y hallazgos en materia de impacto sobre la estabilidad económica y social que, finalmente, genere un efecto en las instituciones democráticas del Estado.

\section{CORRUPCIÓN: DE UN TÉRMINO Y SUS ALCANCES}

Detenerse en el análisis del fenómeno de la corrupción y el escenario variopinto que sus múltiples definiciones plantean, hace imprescindible retomar con anterioridad la figura del Estado y sus instituciones puesto que, como ya se ha planteado, aquella conducta desviada tiene incidencia en la gestión de lo público. Por consiguiente, se torna urgente que los distintos gobiernos unan esfuerzos para hallar su solución a fin de fortalecer su legitimidad de cara a la ciudadanía. Sobre este asunto, Anderson y Teverdova (2003), citados en Cosano (2016), señalan que la corrupción está relacionada con la erosión tanto de las instituciones como de la legitimidad política.

Una primera definición al respecto, quizá bastante general, es otorgada por Díaz (2015) toda vez que considera al Estado como "un orden jurídico de convivencia que organiza y combina elementos sociales complejos en aras de servir a la sociedad" (p. 38). Desde otra posición, el Estado sustenta una comunidad política basada en intereses colectivos, condición que marca un límite con otras organizaciones sociales tales como la familia (Díaz, 2015). En ambos planteamientos se observa una similitud alrededor de la idea organizativa del Estado, lo social como lo colectivo y la convivencia como principio angular de la vida en sociedad. Bajo este sentido, referirse al Estado trae consigo una connotación de lo público, el ejercicio del poder entre las partes involucradas y el acuerdo común de que exista una dirigencia encabezada por ciertas figuras. Ampliando tal conjunto de ideas, Hernández (2009) afirma:

Hay dos conceptos de Estado: uno identifica al Estado con el aparato de poder que organiza y dirige a la población de un determinado territorio; otro identifica al Estado con la población que vive en ese territorio, o sea, con toda la sociedad, que se llama sociedad política porque es a la vez sujeto y objeto de dicho poder. (p. 154) 
La interposición del concepto de poder en la relación gobernantes-gobernados que ejemplifica el Estado atañe, entre otras cosas, a la legitimidad. Esta se define como aceptación del pueblo sobre la forma en que los gobiernos dirigen, y se expresa en las garantías que desde el aparato estatal se les brinda. La legitimidad, más allá de esto, está circunscrita a la validez y vigor que la parte gobernada otorga a sus gobernantes y que, en últimas, pasan a describir la relación entre ambos extremos (Jurado, 2000). Esta idea se amplía en Badía (1971) quien observa, sobre la base de una armonía social en convivencia, el camino claro para la reciprocidad entre el hombre y su ideal de sociedad, así como de la sociedad como tal. Si nos centramos en ello, las demandas que se le hacen al Estado en cuestión de brindar garantías y modificar su comportamiento hacia la protección de lo público en función de lo colectivo son cada vez mayores. En este contexto, afirma Jurado (2000): "el Estado moderno ha adquirido una enorme cantidad de responsabilidades que si bien aumentan significativamente su potencialidad, también incrementan en una proporción mayor aún las expectativas de la sociedad hacia sus actividades" (p. 130). En consecuencia, las garantías que brinda el cuerpo estatal, desde sus instituciones y la legitimidad otorgada a ellas, descansan en una relación proporcional.

En otro orden de ideas, actualmente, los términos de legitimidad y Estado son ampliamente cercanos a la democracia como forma específica de gobierno. Pareciese que la discusión sobre un modelo alternativo se hubiera agotado y que, en últimas, la legitimidad constitutiva como adecuación del proceso de elección de las autoridades públicas al régimen legal (Jurado, 2000) estuviera descartada —al respaldarse por el modelo democrático - y se considere más relevante el análisis de la legitimidad funcional. Sobre esta reza Jurado (2000): "es aquella que cotidianamente tiene que realizar un gobierno durante toda su existencia: consiste en alimentar los principios de legitimidad en que se apoya a través del desempeño eficiente y satisfactorio de las tareas que tiene asignadas" (p. 137). En adición a esto, Mariani (2006), citado en Seligson (2008), cuestiona la relación intrínseca entre democracia y legitimidad toda vez que se enfoque únicamente su tipología constitutiva, por ello afirma que

la democracia no es sólo [sic] un principio de legitimación de gobiernos mediante elecciones, sino también un principio de organización de la sociedad mediante la vigencia de un sistema legal que se base en el reconocimiento de la igualdad de todos los individuos y un poder político e instituciones que procuran garantizar las condiciones de esa igualdad o, en otros términos, del goce universal de los derechos que ese sistema legal consagra. (p. 190)

Como resultado de este análisis puede concluirse que el Estado, como modelo organizativo de las sociedades humanas por ulterior excelencia, yace sobre un acuerdo común en el que dos partes se encuentran involucradas. Mientras que los gobernados establecen las exigencias para mantener esta condición, los gobernantes deben garantizar, en teoría, tales peticiones toda vez que su cumplimiento 
u omisión repercute en la relación legítima de ambos extremos. Alrededor de una idea más procedimental, tal legitimidad encuentra único fundamento, actualmente, en la democracia como forma de gobierno; es en ella donde la libertad de elección del ser humano encuentra apogeo y protección legal. No obstante, la percepción de legitimidad alberga un sentido práctico vislumbrado en el quehacer del Estado y sus instituciones, quehacer desplegado en el poder sobre lo público.

En aras de seguir el orden lógico planteado, de acuerdo con Rúa (2013) son muchas las ocasiones en que ciertos fines particulares, contenidos en la élite política de un país, son superpuestos a los intereses generales de la población y su cumplimiento como fin último del Estado. Así pues, el aprovechamiento de una posición política dominante, cuyo fin sea cumplir intereses personales o de un grupo selecto en vez del bien común o de la mayoría, causa degeneraciones en esa forma de gobierno democrática.

Desde luego, el fenómeno de la corrupción no solo se ha vuelto, en los últimos años, presa de los medios de comunicación, sino que también, en palabras mayores, ha provocado un desajuste en la forma en que se concibe hacer política en tanto perpetúa un imaginario desagradable de la sociedad hacia ella, lo que propicia una mayor crisis de los modelos democráticos (Rollón y García, 2019). Sobre esta línea de ideas, Mendieta (2002) observa en la corrupción una manera de incumplir los deberes incluidos en el quehacer público, al igual que el abuso de confianza tras su desarrollo, lo que comporta una mirada politológica superpuesta a una mirada económica, jurídica o sociológica. Por lo tanto, bajo este enfoque gobernativo, se considera "la corrupción como un síntoma de debilidad institucional o de inadecuados y/o ineficientes marcos normativos para controlar comportamientos desviados, o como expresión de entramados institucionales que facilitan el abuso de poder" (Rollón y García, 2019, p. 6). En efecto, todos estos factores, si bien se plasman desde lo abstracto como lo es la legitimidad del mandato de un gobierno o el nivel de aceptación que dictan las encuestas de una política en específico, su materialización está supeditada al mal uso que se le da a lo público. Ejemplo de ello puede ser la malversación de fondos, robo inescrupuloso del fisco, aventajamiento de la posición en el sector público para obtener o brindar favores a cambio de algún tipo de retribución, entre otros muchos.

De acuerdo con las cifras y como ya había sido planteado, el análisis de la corrupción deviene en dos sentidos. Uno de ellos, acogiendo la medición alrededor de cómo se percibe este fenómeno en cuanto objeto de estudio, se presenta en Latinobarómetro (2018) al afirmar que mientras en el año 2017 un 62 \% de la población encuestada percibía la corrupción como un fenómeno en aumento, para el 2018, un año más adelante, la cifra aumentó al $65 \%$. Concorde a esto último y sobre casos más específicos, se afirma: "El mirar la corrupción como problema principal del país por país, vemos los tres países que están en primer lugar, Colombia con 
20 \%, Perú 19 \%, Brasil 16 \% y México con 14 \%." (Latinobarómetro, 2018, p. 59). Al detenerse en la severidad de los datos que nos presenta tal encuesta sobre cuáles fenómenos sociales son mayormente percibidos en el caso colombiano, la corrupción, competencia de esta investigación, encabeza tal podio.

Dicha percepción encuentra fundamento sobre el estudio anual de Transparencia Internacional que encamina su actuar sobre la segunda vertiente de análisis, tal y como encuentra lugar en la medición de la corrupción a través del actuar institucional. En una escala de 0 a 100, donde el número más bajo indica un índice mayor de corrupción y la cercanía al número más alto supone un índice menor de la misma, Colombia obtiene una puntuación de 37 y su escalafón, entre los países más corruptos, es de 96 entre 180 (Transparency International, 2020). Con ambos estudios en mente y la serie de afirmaciones que existen entorno a la corrupción, se plantean las siguientes preguntas: ¿Qué debe entenderse como corrupción? ¿Cuáles son sus elementos definitorios? ¿Cuál es su clasificación? En pesquisa de ello se levantan definiciones teóricas que, al fin y al cabo, alimentan el corpus legal sobre el cual tal conducta halla una sanción.

Someros acercamientos como el que hace Díaz (2003) al afirmar que "la corrupción está referida a conductas violatorias de acuerdos sobre lo común, lo que es de todos, lo público" (p. 145), vislumbran la esfera que se ve afectada principalmente y, de manera simultánea, pone entre líneas el establecimiento de lo que en sociedad se ha previsto como apto. Este mismo sentido se ve expresado en Isaza (2012), quien determina como conducta corrupta "un acto que viola reglas de comportamiento, que perjudica los recursos públicos y el interés general y que produce una ganancia privada, la cual puede ser de diversos tipos: monetaria, de posición, política, de información privilegiada, entre otras" (p. 221) . Bajo el mismo conjunto de ideas, el Consejo Nacional de Política Económica y Social [Conpes] (2013), en su documento 167, define la corrupción como "el uso del poder para desviar la gestión de lo público hacia el beneficio privado" (p. 18). Frente a ello, estas tres primeras definiciones albergan como puntos en común la afectación de lo público y la superposición de lo privado sobre el interés general, a partir de una trasgresión de las reglas de juego aceptadas socialmente.

En consideración a esto, la definición planteada por el Conpes incluye el "uso del poder" como método a través del cual se llevan a cabo actos corruptos. Este elemento, sin duda alguna, determina la posición que la parte involucrada tiene en el aparato estatal y cómo saca provecho de ella. De esta manera, como se verá a continuación, tiene un rol angular en definiciones teóricamente más complejas.

Un ejemplo lo refleja Kramer (1977), citado en Pont y Arango (2017), toda vez que denota en la corrupción un comportamiento exclusivo de los funcionarios públicos el cual, en su ejercicio, diverge de lo formalmente establecido como deber para servir a fines particulares o privados. Del mismo modo, Thompson (1993) "describe la corrupción convencional como aquella situación en la que un funcionario público gana, 
un ciudadano recibe un beneficio, y la conexión entre la ganancia y el beneficio es inapropiada, pues corresponde a lo que él llama un 'motivo corrupto'" (citado en Pont y Arango, 2017, p. 37). A manera de síntesis, Pont y Arango (2017) afirman: "Todas estas definiciones coinciden en que se trata de un comportamiento que abusa o se desvía de los deberes formales de un rol público/poder público/cargo público, para obtener un beneficio o ganancia privada (personal o para un tercero)" (p. 35). El universo de estas perspectivas, si bien agrega al funcionario público como aquel hacedor del acto corrupto al utilizar su posición de poder en la relación asimétrica con el ciudadano y que, dependiendo de su rango en la misma, obtiene mayor provecho o no, es indiferente a los escenarios donde puede presentarse y la cantidad de agentes que participan.

En comparación con tales definiciones, si bien más elaboradas y concisas que las primeras, un tercer grupo identifica las esferas del poder estatal y la pluralidad de involucrados que se hace manifiesta en la cadena del fenómeno de la corrupción. En relación con esto, Valdez (1997), citado en Pont y Arango (2017), plantea:

[la corrupción se define en] la violación limitada de una obligación por parte de uno o más decisores con el objeto de obtener un beneficio personal extra posicional del agente que lo(s) soborna o a quien extorsiona(n) a cambio del otorgamiento de beneficios para el sobornante o el extorsionado que superan los costos del soborno o del pago o servicio extorsionado. (p. 36)

Tal comportamiento simultáneo en más de uno o dos decisores — destacada en la anterior cita - dan una mejor entrada hacia la definición que plantea Arvind (2001) cuando ve en la corrupción "aquellas actividades en las que los funcionarios públicos, los burócratas, los legisladores y los políticos utilizan poderes delegados a ellos por el público para promover sus propios intereses económicos a expensas del bien común" (p. 73). Con base en esto, la especificidad sobre quienes recae la conducta y lo definitorio de la conducta en sí, logra gestarse en los tres grupos de perspectivas sobre la corrupción descritos: i) como guía específica y esencia de la corrupción; ii) con la inclusión de la relación de poderes representada en el poder estatal que habla de los actores involucrados prioritariamente en la conducta; $y$ iii) dilucida los escenarios donde puede llevarse a cabo y el total de individuos que pueden llegar a realizarla.

A partir de estos elementos, Shah y Schacter (2004) enuncian tres tipos comunes de corrupción independientes a su tipificación en cualquier marco jurídico de los Estados, los cuales son: i) pequeña corrupción administrativa o burocrática (petty administrative or bureaucratic corruption); ii) gran corrupción (grand corruption); y iii) captura de Estado/ tráfico de influencias (state capture/influence peddling). En ese orden, la primera tipificación corresponde a las transacciones aisladas que realizan funcionarios públicos en la lógica de aprovechar su posición. En segundo lugar, la gran corrupción se define por el robo o mal uso de grandes cantidades de recursos públicos por actores oficiales de la élite administrativa. En últimas, la captura de Estado y tráfico de influencias, que ocupa una mayor jerarquía, trata las veces o el escenario en que el sector privado 
captura cualquiera de las esferas del aparato estatal (legislativa, ejecutiva o judicial) en su beneficio (Shah y Schacter, 2004).

En este hilo de ideas, las posturas teóricas entorno al concepto de corrupción a gran escala, vale aclarar en su sentido literal, no arrojan muchos resultados. Estudios realizados y plasmados en español sobre la materia se reflejan en Transparencia Internacional (2009), Botero y Juárez (2015), Martini (2015), García (2016), Sánchez (2017) y Suárez (2018); todos ellos son fundamentales a la hora de hacer un análisis ontológico sobre el fenómeno. Sin embargo, el ejercicio se enriquece toda vez que la búsqueda en bases de datos académicas, a partir de las traducciones más cercanas del concepto en inglés high corruption o large-scale corruption son exitosas y se enmarcan en la tipología ya mencionada de gran corrupción (grand corruption). Sobre esta definición, las investigaciones son más exhaustivas en español e inglés, por ese motivo tomamos como base a Elliott (1997), Amundsen (1999), Unodc (2004), Gopac (2013), Transparency International (2015) y Guzmán y Ponce (2017). En cada una de ellas se encontraron, como se verá a continuación, elementos de los que bebe la corrupción a gran escala que posibilitan una definición más sólida al respecto.

Con base en esto, la gran corrupción (GC de ahora en adelante) se caracteriza por "tomar lugar en los niveles más altos del sistema político. Es cuando los políticos y agentes del Estado, quienes tienen derecho a realizar y hacer cumplir las reglas en el nombre del pueblo, son corruptos en sí" (Amundsen, 1999, p. 3). Alrededor de esta precisión se identifican dos aspectos esenciales. En primer lugar, se detecta la escala jerárquica del sistema político en donde es desarrollada; el sistema político, en este caso, abre las puertas a un fenómeno que, así como puede tener desarrollo en el sector administrativo, también puede hacerlo en el legislativo o judicial. Esta idea se complementa, más allá de encontrar específicamente a miembros de la política o funcionarios del Estado como hacedores de la corrupción, con la posición que ocupan con respecto al desarrollo de la norma, bien sea de creación o de encargarse de su cumplimiento. Quizá no hay mejor modo de cerrar esta distinción que en palabras del mismo autor, ya que cuando aparta la gran corrupción de la pequeña corrupción, si bien ambas tienen lugar en el sector público, la segunda únicamente encuentra despliegue en la Administración (Amundsen, 1999).

Una distinción similar se halla en Unodc (2004) —siglas que en español se refieren a la Oficina de las Naciones Unidas contra la Droga y el Delito- quien explica que la GC perversa los altos niveles de un gobierno y trae consigo la erosión de la confianza en sus actividades, el imperio de la ley y la estabilidad económica. Vale la pena resaltar esta última consecuencia, ya que involucra el elemento monetario como una de las formas de beneficios en la práctica corrupta. En adición, los casos de GC que involucran altas cantidades de dinero plantean, por lo general, saqueos sistemáticos realizados por funcionarios del Gobierno o del tesoro público (Unodc, 2004, p. 29). Lo 
importante de esta perspectiva, además de acentuar los actores que prevalecen en la GC, es su incidencia en la economía de un país a expensas de intereses particulares.

Como un acercamiento más sintetizado a la GC, Gopac (2013) — que por sus siglas al español refieren a la Organización Global de Parlamentarios Contra la Corrupciónidentifica en los políticos y los agentes del Estado sus actores principales. No obstante, menciona que la finalidad última es "mantener su poder, su estatus y su riqueza. Esencialmente, la gran corrupción no únicamente viola las leyes nacionales, sino lo que es aún más grave, distorsiona y menoscaba el estado de derecho en sí" (Gopac, 2013, p. 3). Bajo este mismo planteamiento, afirma que una definición rigurosa de la GC responde a cinco preguntas: i) categoría de personas involucradas en los delitos de GC; ii) criterios para determinar el rango de "alto nivel"; iii) tipo de beneficios e incidencia/cantidad que son puestos en juego; iv) alcance de la GC sobre la dignidad humana; y v) nivel de sistematicidad de los daños realizados a través de GC. Sobre estas cuestiones nos centraremos más adelante para intentar construir una definición de la corrupción a gran escala.

Situándonos en el valor agregado que da Transparency International (2015) al abordaje de la GC, observa en ella un "gran obstáculo para alcanzar el desarrollo sostenible. (La GC) socava y distorsiona la actividad económica y la distribución de recursos, [...] arraigando la pobreza e incrementando la exclusión" (p. 1). Con esto dicho, la GC, aparte de tomar distintas formas de ejecución — sea piramidal o de una solo momento-, resulta en una violación de los derechos humanos, donde prima el beneficio de unos pocos sobre el de muchos (Transparency International, 2015). Un ejemplo de esto se prioriza en Guzmán y Ponce (2017) quienes analizan la incidencia de la GC en la prestación de servicios públicos en México; con este fin determinan:

La gran corrupción suele estar presente cuando la agencia gubernamental estructura el presupuesto y gasta los recursos. Puesto que la gran corrupción tiende a desviar cantidades importantes de dinero, es probable que termine por fortalecer la asociación que los ciudadanos establecen entre la corrupción y una provisión menor del servicio. (pp. 244-245)

En referencia a lo anterior, la vulneración de un servicio público por un acto corrupto, sistemático y concertado deriva en afecciones a la calidad de vida y la misma dignidad humana. De ahí que las garantías que el Estado como ente organizacional debe brindar son discutidas para dar paso a un mayor descontento y probabilidad de que el sistema se torne disfuncional. Por último, la revisión de Elliot (1997), si bien altera el sentido cronológico establecido, no está desprovista de lógica alguna. Para ella, la GC tiene lugar únicamente en aquellas decisiones de gobierno que, sin un alto involucramiento político, no podrían realizarse o tomarse. En este sentido afirma Elliot (1997): "Es en los altos niveles de gobierno, donde los líderes políticos, la burocracia, y el sector privado, todos interactúan" (p. 178). En este estudio se plantea un nuevo actor que, si bien se daba por sentado como 
recurrente en los actos de corrupción "menores", no es mencionado con prioridad en las definiciones alrededor de la GC, estos son los agentes del sector privado.

Según las características de los actores involucrados, cada una de ellas escala en una clase distinta de corrupción que, para efectos de este estudio, denominaremos microcorrupción, mesocorrupción y macrocorrupción. En resumen, la microcorrupción encuentra un equivalente con la pequeña corrupción y se evidenciaría bajo montos mínimos de dinero involucrados en el acto sobre acciones realizadas por miembros de una baja jerarquía, especialmente de la burocracia. Un segundo momento lo ocuparía la mesocorrupción, espacio con un mayor desarrollo de influencias que engrandecen el acto corrupto y, por ende, su implicación en la calidad de vida de los ciudadanos o miembros de un sector en específico es mayor. Como pico de la escala, encontramos la macrocorrupción. Sobre esta no hay novedad que ya haya sido desarrollada en la GC, pero al ser abordada desde esta clasificación jerárquica, da paso a una lectura del fenómeno en torno a la complejidad de sus partes, la interacción entre ellas y los resultados que desde allí pueden obtenerse. Para un mejor efecto, la figura 2 plasma lo dicho.

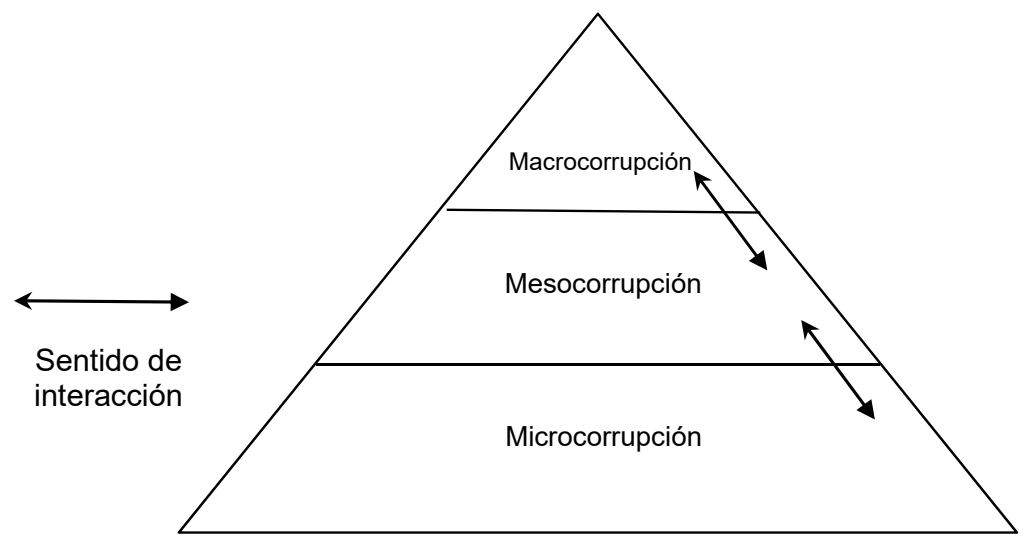

Figura 2. Jerarquía piramidal de la corrupción

Fuente: elaboración propia.

Como es posible observar, la interacción entre las distintas jerarquías o "pisos" de la corrupción connota una dinámica bidireccional. La explicación se puede dar de las siguientes formas:

1. Todas las dimensiones están conectadas entre sí. Un caso de microcorrupción (M1) puede suscitar el ascenso jerárquico de un funcionario o un actor político en el Estado al nivel medio (que incluso puede enfocarse en uno alto). De esta manera, tiene participación en la mesocorrupción y ensancha un juego de compromisos adquirido entre ellas. 
2. Desde la mesocorrupción (M2), el tráfico de influencias, que por excelencia la describe, funge como escala pivote, toda vez que puede boicotear en una mayor medida la posición de los agentes del Estado o lograr su ascenso a la escala máxima.

3. El pico de la pirámide lo obtiene la macrocorrupción (M3). Desde allí, al saber que imperan las grandes esferas del poder Estatal, se puede desarrollar una cadena de influencias cuesta abajo que, en una serie de juego de favores y compromisos adquiridos, alimentan la corrupción como factor integral del sistema.

Está claro que en cada una de las jerarquías se hace un daño indirecto o directo al bien público en mayor o menor medida. De todo esto se infiere que, aunque la corrupción sea en términos económicos, otras instancias trascienden en la jerarquía misma del Estado y transforman su esencia en un vehículo corrupto.

Lo analizado sobre la corrupción a gran escala (CAGE de ahora en adelante), en un sentido literal al español, no arroja mayores luces que lo ya visto sobre GC. Ureña (1997), citado en Botero y Juárez (2015), desarrolla un sistema de colores para la corrupción: en este caso, la CAGE es catalogada como corrupción negra, en la que "participan funcionarios de alto estatus" (p. 6). Esta definición flexibiliza, aún más, los involucrados y la esfera desde la que pueden actuar.

En consecuencia, si bien los insumos desde la GC alimentan la definición de CAGE, es tarea importante sintetizarlos bajo esta última tipología y ampliar la secuencia de resultados que puedan obtenerse. Además, como se verá más adelante, es menester señalar la urgencia de que sea tipificada en el ordenamiento jurídico colombiano y los retos que se le han venido planteando en los últimos tiempos desde casos internacionales. Así, la CAGE es un acto corrupto desarrollado por agentes de alto nivel que están activos o tienen participación latente en el sistema político. En este lugar no interesa su rol — sea funcionario público, político o actor privado—a través del cual se busque alcanzar un beneficio económico, de influencias o favores y que, al alterar el orden de las reglas en el Estado de derecho y la democracia, no permite una óptima distribución de los recursos, los servicios y las oportunidades como garantías obligatorias a brindar por el Estado. De esta corrupción deriva la denigración, afectación de la calidad de vida y el desarrollo sostenible del pueblo y generaciones venideras, además de enquistar todo el contexto socioeconómico y político en una conducta desviada del deber ser de las cosas.

\section{EL MÉTODO DE ESCENARIOS COMO HERRAMIENTA PARA LA IDENTIFICACIÓN DE LA NOCIÓN DE CORRUPCIÓN A GRAN ESCALA EN EL ORDENAMIENTO COLOMBIANO}

Como se determinó en el acápite que antecede se pueden encontrar tres tipos de corrupción que responden a una organización jerárquica: micro, meso y macrocorrupción. En este sentido, atendiendo a las especificaciones planteadas en 
el apartado metodológico y el porqué de la decisión, en el presente desarrollo solo se tomarán cuatro tipos penales (P1, P2, P5 y P6) sobre los cuales se puede ejemplificar de mejor manera el ejercicio que intenta demostrar la configuración de las distintas corrupciones según su jerarquía, sin implicar una disminución en la importancia que las demás guardan y, a su vez, reconociendo en cada una de ellas un aspecto fundamental en la propuesta teórica establecida. Esto conllevará a identificar si se puede hablar de la CAGE de manera autónoma en el ordenamiento colombiano, o si no hay un tipo penal que se fundamente propiamente en esta noción y se encasillen en ella hay una multiplicidad de tipos al momento de presentarse hechos. Por lo anterior, se debe realizar el análisis de los diferentes tipos categorizados previamente.

\subsection{Peculado}

Este tipo penal responde al hurto de cosas muebles cuyo propietario es el Estado (Franco, 2014). No obstante, parte de la doctrina sostiene que este se entiende como el delito cometido por el funcionario encargado de administrar bienes que son propiedad del Estado o de particulares, pero que se encuentran bajo la administración del Estado. Sin embargo, se apropia de ellos o los usa de manera indebida (Ortega, s.f.; Sanguino Madariaga, 2008; Uribe, 2012). Los tipos penales que se encuentran bajo este precepto son:

Artículo 397. Peculado por apropiación. El servidor público que se apropie en provecho suyo o de un tercero de bienes del Estado o de empresas o instituciones en que éste tenga parte o de bienes o fondos parafiscales, o de bienes de particulares cuya administración, tenencia o custodia se le haya confiado por razón o con ocasión de sus funciones.

\section{$[\ldots]$}

Artículo 398. Peculado por uso. El servidor público que indebidamente use o permita que otro use bienes del Estado o de empresas o instituciones en que éste tenga parte, o bienes de particulares cuya administración, tenencia o custodia se le haya confiado por razón o con ocasión de sus funciones.

\section{$[\ldots]$}

Artículo 399. Peculado por aplicación oficial diferente. El servidor público que dé a los bienes del Estado o de empresas o instituciones en que éste tenga parte, cuya administración, tenencia o custodia se le haya confiado por razón o con ocasión de sus funciones, aplicación oficial diferente de aquella a que están destinados, o comprometa sumas superiores a las fijadas en el presupuesto, o las invierta o utilice en forma no prevista en éste, en perjuicio de la inversión social o de los salarios o prestaciones sociales de los servidores.

$[\ldots]$

En estos se deben identificar los elementos del tipo penal: 
Sujeto activo: servidor público a quién se le confió la custodia, administración o tenencia del objeto razón de sus funciones.

Sujeto pasivo: el Estado.

Objeto jurídico: recto ejercicio de la función pública de administración.

Objeto material: bienes confiados al servidor. (Ortega, s.f.)

\section{Escenario}

Debido a los sujetos que interviene y las acciones necesarias este tipo se ubica en la micro corrupción; ya que no implica la participación de actores terceros a la administración o el giro de sumas de dinero a ellos (figuras 3 y 4).

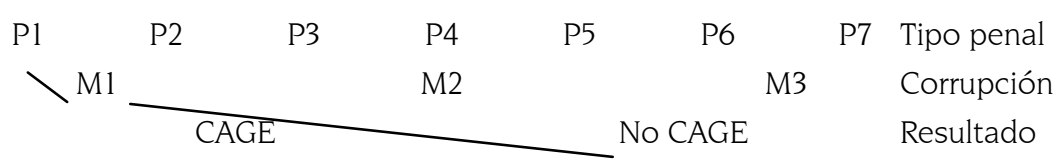

Figura 3. Escenario realizable peculado

Fuente: elaboración propia a partir de Vargas (2017) y Rúa, Duarte y Gómez (2018).

En consecuencia:

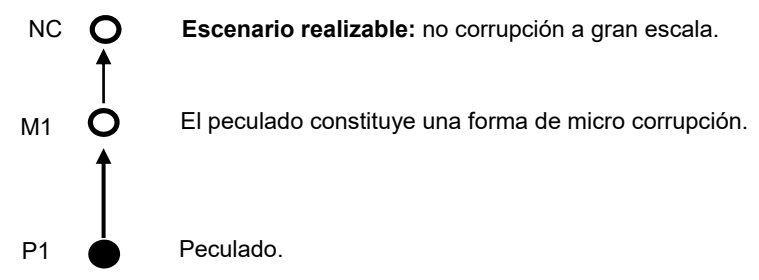

Figura 4. Cadena de razonamiento escenario peculado

Fuente: elaboración propia a partir de Vargas (2017) y Rúa, Duarte y Gómez (2018).

\subsection{Concusión}

Este tipo penal responde a la reclamación arbitraria efectuada por un funcionario en provecho propio (Franco, 2014). Esto significa que la exigencia de prestaciones o utilidades de manera arbitraria y abusiva.

Artículo 404. Concusión. El servidor público que abusando de su cargo o de sus funciones constriña o induzca a alguien a dar o prometer al mismo servidor o a un tercero, dinero o cualquier otra utilidad indebidos, o los solicite, incurrirá en prisión. (Arboleda Vallejo, 2009) 
Es importante exponer que, respecto del objeto jurídico de la concusión, Uribe (2012) lo delimita a la rectitud, fidelidad, transparencia y honestidad a partir de las cuales todo servidor debe desempeñar sus funciones. Para ello, debe abstenerse de obtener lucro diferente a lo percibido por su salario (Uribe, 2012).

En estos se deben identificar los elementos del tipo penal:

Sujeto activo: servidor público a quién se le confió la custodia, administración o tenencia del objeto razón de sus funciones.

Sujeto pasivo: el Estado.

Objeto jurídico: probidad de la actividad estatal y ética en el ejercicio de la función pública.

Objeto material: sujeto a quien induce constriñe y solicita. (Ortega, s.f.)

\section{Escenario}

En consecuencia, debido a los sujetos que intervienen y las acciones necesarias, este tipo de corrupción se ubica en la mesocorrupción, toda vez que la posición del servidor público, además de brindar una ventaja para sí mismo, implica la existencia de un tercero que resulta beneficiado como producto de una posición primera. Conforme a ello, se inicia una interacción transaccional entre la esfera privada y pública que no solo roza con lo monetario, sino también con un ascenso en la escala del poder estatal. De esta manera, la mesocorrupción, como etapa meridional en el fenómeno tratado, hace las veces de un catalizador hacia la macrocorrupción con destino a CAGE, producto del tráfico de influencias como característica esencial (figuras 5, 6 y 7).

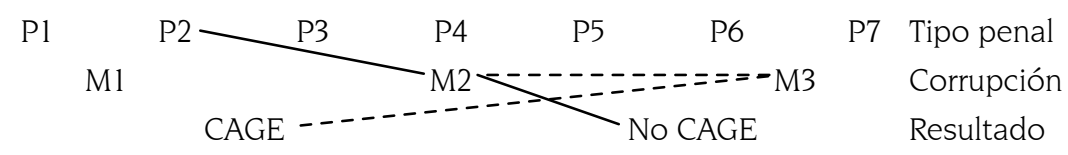

Figura 5. Escenario realizable concusión

Fuente: elaboración propia a partir de Vargas (2017) y Rúa, Duarte y Gómez (2018).

En consecuencia:

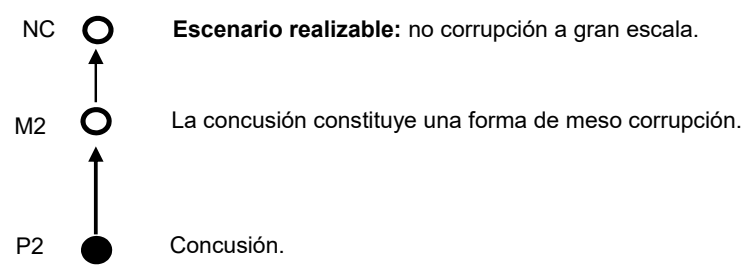

Figura 6. Cadena de razonamiento escenario realizable concusión

Fuente: elaboración propia a partir de Vargas (2017) y Rúa, Duarte y Gómez (2018). 
No obstante:

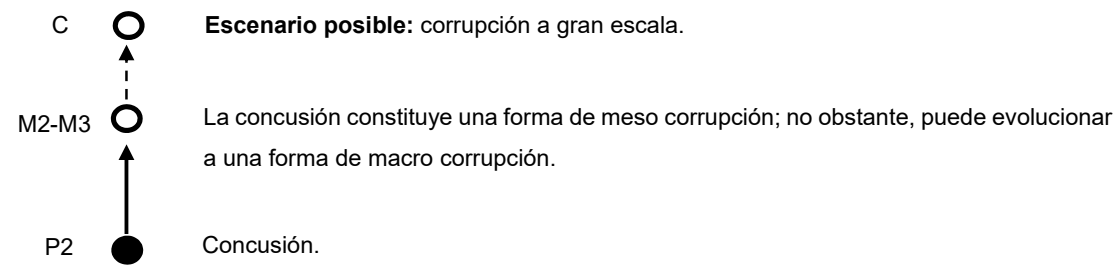

Figura 7. Cadena de razonamiento escenario posible concusión

Fuente: elaboración propia a partir de Vargas (2017) y Rúa, Duarte y Gómez (2018).

\subsection{Tráfico de influencia}

Se encuentra tipificado de la siguiente manera:

Artículo 411. Tráfico de influencias de servidor público. El servidor público que utilice indebidamente, en provecho propio o de un tercero, influencias derivadas del ejercicio del cargo o de la función, con el fin de obtener cualquier beneficio de parte de servidor público en asunto que éste se encuentre conociendo o haya de conocer.

$[\ldots]$

En este se debe identificar los elementos del tipo penal:

Sujeto activo: servidor público dentro de su competencia de aprobación y celebración del contrato estatal.

Sujeto pasivo: el Estado.

Objeto jurídico: igualdad de los ciudadanos frente a la administración e imparcialidad.

Objeto material: influencias que el agente posee por razón de su cargo o función. (Ortega, s.f.)

\section{Escenario}

El tráfico de influencias destaca, entre los ya mencionados delitos, un primer momento donde la CAGE logra configurarse gracias a los sujetos que se ven involucrados. La explicación, no obstante, puede tomar dos caminos. El primero de ellos, una vez se gesta la mesocorrupción, no da lugar a la CAGE, pero, según quienes tengan parte en el delito, encontraría ocasión la macrocorrupción. En este sentido y bajo una dinámica progresiva del delito, podría configurarse un caso de macrocorrupción, etapa última y proyectada hacia la CAGE. La variabilidad de la clasificación encuentra como piedra angular los sujetos que entran a participar y la misma posición en el sector público que posee el funcionario. Este es el motivo principal por el cual el privado guarda para interactuary postergar la relación corrupta entre ambas partes (figuras 8, 9y 10). 


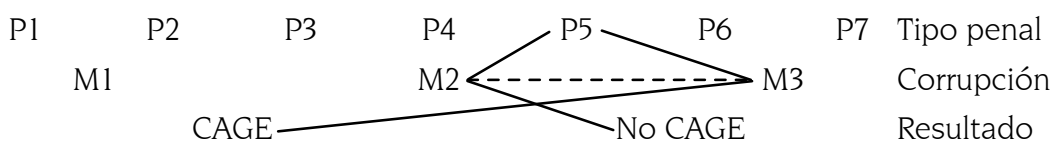

Figura 8. Escenario realizable tráfico de influencia

Fuente: elaboración propia a partir de Vargas (2017) y Rúa, Duarte y Gómez (2018).

En consecuencia:

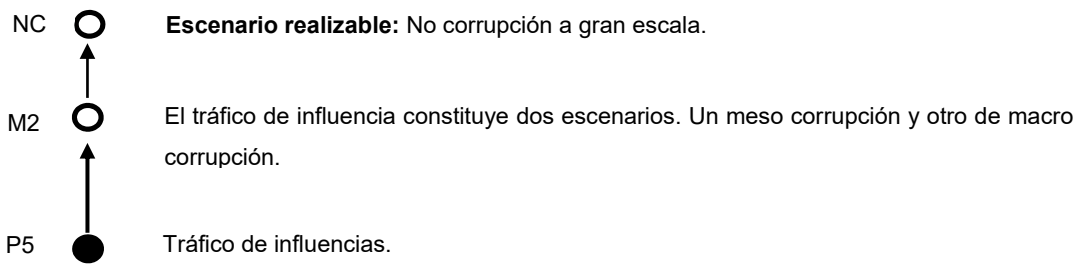

Figura 9. Cadena de razonamiento escenario realizable tráfico de influencia

Fuente: elaboración propia a partir de Vargas (2017) y Rúa, Duarte y Gómez (2018).

No obstante:

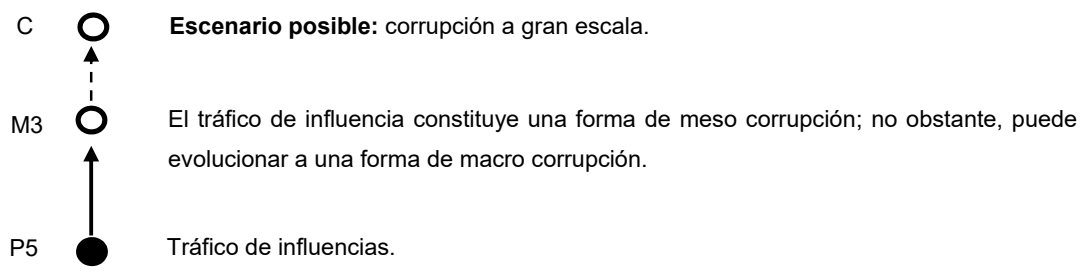

Figura 10. Cadena de razonamiento escenario posible tráfico de influencia

Fuente: elaboración propia a partir de Vargas (2017) y Rúa, Duarte y Gómez (2018).

\subsection{Enriquecimiento ilíito}

La Corte Constitucional, en la Sentencia C-319 (1996), ha manifestado que el enriquecimiento ilícito constituye un delito cuya autonomía tiene origen en la Constitución y no puede entenderse como subsidiario. El tipo penal hace referencia al bien jurídico orden económico y social. Tiene por objeto proteger las condiciones que surgen de un orden económico y social derivado del trabajo y los contratos (Sanguino Madariaga, 2008; Fiscalía, 2018). El tipo penal expone:

Artículo 412. Enriquecimiento ilícito. El servidor público, o quien haya desempeñado funciones públicas, que durante su vinculación con la administración o dentro de los cinco (5) años posteriores a su desvinculación, obtenga, para sí o para otro, incremento patrimonial injustificado. 


\section{$[\ldots]$}

En este se debe identificar los elementos del tipo penal:

Sujeto activo: servidor público dentro de su competencia de aprobación y celebración del contrato estatal.

Sujeto pasivo: el Estado.

Objeto jurídico: propiedad y honestidad de la administración pública.

Objeto material: incremento patrimonial en bienes o activos de cualquier especie. (Ortega, s.f.)

\section{Escenario}

La clasificación de este delito, conforme a los sujetos que toman participación y el orden que se ve afectado, tal como lo es el económico y social, tiene lugar en la macrocorrupción. El incremento del patrimonio de manera ilícita se da a expensas de la posición privilegiada que guarda el funcionario público y la capacidad que, desde ella, tiene para la celebración de contratos o el desarrollo de acciones que brinden una mayor ventaja. Partiendo de esto, es posible tratar una CAGE al verse materializado, tras ello, el tráfico de influencias, el perjuicio al patrimonio público y el debilitamiento del entorno económico y social; en cuanto que las acciones del Estado se verían permeadas por un interés más particular que general. De la misma manera, se habría de configurar un daño en abstracciones tales como la confianza y la legitimidad de las instituciones públicas, lo cual no influyendo solo en las acciones públicas, sino también en el mismo sistema (figuras 11 y 12).
P1
P2
P3
P4
M2
$\stackrel{P 5}{P 6} \coprod_{M 3}$
M1
CAGE
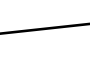
No CAGE
P7 Tipo penal
Corrupción
Resultado

Figura 11. Escenario realizable enriquecimiento ilícito

Fuente: elaboración propia a partir de Vargas (2017) y Rúa, Duarte y Gómez (2018).

En consecuencia:

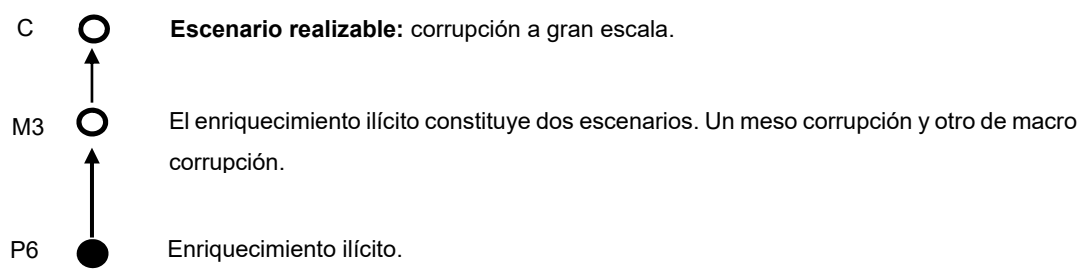

Figura 12. Cadena de razonamiento escenario realizable enriquecimiento ilícito

Fuente: elaboración propia a partir de Vargas (2017) y Rúa, Duarte y Gómez (2018). 


\section{CONCLUSIONES}

La corrupción como fenómeno social surge de la interacción entre el Estado y particulares, y trasciende la escala privada o pública. Sobre esta afirmación, su complejidad descansa en lo multidimensional de la conducta una vez está determinada por la confluencia y relación de diferentes variables del orden social. Así, insta a su comprensión e interpretación de acuerdo con el contexto en que se presenta. Una recopilación de lo presentado tiene inicio en el análisis del Estado como figura en la que se concentran bandos - entiéndase gobernantes y gobernados_ cuyo principio de aceptación y legitimidad reposa en el quehacer de las instituciones al igual que su actuación. Presentada la corrupción desde un sentido más general, la desviación de la conducta del servidor público que atenta el bienestar de la sociedad en aras de lograr satisfacer un interés particular plantea un entredicho hacia el comportamiento del Estado y sus entidades regulatorias; para el caso, el sistema judicial.

Ahora bien, realizado un ejercicio bajo una dinámica de distinción entre las distintas modalidades de corrupción y la corrupción a gran escala, se postula esta última desde un sentir más amplio. En este sentido, se atribuye la participación al sector privado donde interactúan otros varios actores, lo cual compromete, a través de sus resultados, el ejercicio de los derechos fundamentales de la sociedad, así como su capacidad para acceder a bienes y servicios. De allí que una revisión de la norma acerca de la tipificación del delito, con saldo negativo, despierta el más amplio sentido crítico y de urgencia sobre el estudio de la corrupción a gran escala.

La consideración metodológica desarrollada advierte escenarios en los que la conducta, primeramente buscada a regular, queda por fuera de lo ya establecido por motivo de la varianza del comportamiento social. De cara a ello, la tarea del Estado debe ser enfocada en reevaluar las conductas reguladas y buscar reintegrarse a una legitimidad funcional que, para la ocasión, deviene del acceso a la justicia.

Sobre el caso colombiano, a razón de lo argumentado, tanto el enriquecimiento ilícito, la concusión y el tráfico de influencias logran comportar escenarios realizables de corrupción a gran escala dadas las características funcionales del delito, en atención asimismo de los actores involucrados según establecida la dinámica de su participación. Por tal motivo, las nociones teóricas son adheridas al entendimiento de una conducta que trasciende lo tipificado y que dista de lo ya establecido.

En consecuencia, el asunto tratado sigue presentándose como un tema de basta y rigurosa definición debido a las categorías y situaciones que se ven involucradas. De esta manera, se desdibuja constantemente la frontera legal que puede existir entre un delito y otro. Al contrario de reducir las motivaciones para afrontar a la corrupción a gran escala como un fenómeno latente y de gran magnitud en Colombia, la amplitud de la materia no solo despierta un sentido crítico desde instancias judiciales, sino 
también por la significancia política, social y económica que en ella confluyen. Del mismo modo, la operatividad del método de escenarios hace replicable la adaptación del estudio a otros países, lo que convierte los resultados de la academia en insumos de gran importancia para esferas gubernamentales o con capacidad decisoria, flageladas en demasía o no por la conducta.

\section{REFERENCIAS}

Amundsen, I. (1999). Political Corruption: An Introduction to the Issues. Michelsen Institute Development Studies and Human Rights.

Arboleda Vallejo, M. (2009). Código penal y de procedimiento penal anotado (Vigesimaquinta ed.). Editorial Leyer.

Arvind, J. (2001). Corruption: A review. Journal of Economic Surveys, 15(1), 71-121. https://doi. org/10.1111/1467-6419.00133

Badía, J. (1971). Poder y legitimidad. Estudios, (180), 5-28.

Botero, A. y Juárez, A. (2015). Percepciones sobre la corrupción de habitantes de Manizales y Pereira. Papel Político, 20(1), 1127-146. https://doi.org/10.11144/Javeriana.papo20-1.pchm

Carnelutti, F. (2013). Cómo nace el derecho. Editorial Temis.

Cely, A. (1999). Metodología de los escenarios para estudios prospectivos. Revista de Ingeniería e Investigación, (44), 26-35. https://revistas.unal.edu.co/index.php/ingeinv/article/view/21 296/22265

Corte Constitucional (1996, 18 de julio). Sentencia C-319 (Vladimiro Naranjo Mesa, M. P.).

Consejo Nacional de Política Económica y Social, Conpes (2013, 9 de diciembre). Conpes 167. Estrategia Nacional de la Política Pública Integral Anticorrupción. https://www.funcionpublica.gov.co/eva/ gestornormativo/norma.php?i=83097

Cosano, P. (2016). La lucha contra la corrupción. En J. Marco Marco y M. Pérez Gabaldón (Coords.), Radiografiando la democracia: un estudio sobre corrupción, buen gobierno y calidad democrática (pp. 10-22). Avapol.

Díaz, C. (2015). Sistema político y formas de gobierno. En X. Arango Morales y A. Hernández Paz (Coords), Ciencia Política Perspectiva Multidisciplinaria (pp. 29-49). Tirant lo Blanch.

Díaz, Á. (2003). Ética y Corrupción. Lo público y la democracia. Convergencia. Revista de ciencias sociales, 10(31), 141-151. Redalyc.Ética y corrupción. Lo público y la democracia

Elliott, K. (1997). Corruption as an International Policy Problem: Overview and Recommendations. En K. Elliot (Ed.), Corruption and the Global Economy, (pp. 175-233). Institute for International Economics.

Fiscalía General de la Nación. (2018). Tipologías de corrupción en Colombia: Tráfico de influencias, enriquecimiento ilícito y otros delitos. Tomo 4. Fiscalía General de la Nación - Unodc.

Franco, J. (2014). Delitos contra la administración pública: una mirada desde la causal novena del principio de oportunidad. Universidad Autónoma Latinoamericana. http://repository.unaula.edu.co:8080/ bitstream/123456789/247/1/unaula_rep_pre_der_2014_delitos_administracion.pdf

Gallup Colombia (2019, febrero). Gallup Poll. Gallup Colombia S.A.S. 
García, E. (2016). Gran corrupción: estrategias para evitar su impunidad internacional. Revista Nuevo Foro Penal, 12(87), 60-98. Gran corrupción: estrategias para evitar su impunidad internacional Dialnet (unirioja.es)

Gopac. (2013). Juzgando la Gran Corrupción como delito internacional. Gopac.

Gutman, P. (1995). Escenarios ecológicos. En G. Gallopín (Ed.), El futuro ecológico de un continente: Una visión prospectiva de la América Latina, (p.1126). United Nations University Press. Fondo de Cultura Económica.

Guzmán, J. y Ponce, A. (2017). Los tipos de corrupción y la satisfacción con los servicios públicos. Evidencia del caso mexicano. Región y Sociedad, 29(70), 231-262. https://doi.org/10.22198/ rys.2017.70.a344

Hernández, J. (2009). El concepto de legitimidad en perspectiva histórica. Cuadernos Electrónicos de Filosofía del Derecho, (18), 153-166. https://dialnet.unirioja.es/servlet/articulo?codigo=3022499

Isaza, C. (2012). El fracaso de la lucha anticorrupción en Colombia. Opera, (11), 221-239.

Jurado, R. (2000). Fundamentos de la legitimidad. Estudios Políticos, (24), 129-153.

Latinobarómetro. (2018). Informe 2018. Corporación Latinobarómetro.

Londoño, L. y Marín, J. (2002). Metodología de la investigación holística: Una propuesta integradora desde las sociedades fragmentadas. Uni-pluri/versidad, 2(3), 22-23.

Maqueda, J. (1996). Cuadernos de dirección estratégica y planificación. Ediciones Díaz de Santos.

Martini, M. (2015). Combatir la corrupción a gran escala: desafíos y éxitos. Transparencia Internacional.

Mendieta, M. (2002). Ética Pública y corrupción en el inicio de un nuevo milenio. Foro Internacional, 645-682.

Ortega, J. (s.f.). Delitos contra la administración pública [Ponencia]. Escuela Superior de Administración Pública. Departamento Administrativo de la Función Pública. https://www.funcionpublica.gov. co/eva/admon/files/empresas/ZW1wcmVzYV83Ng==/archivos/1463963379_35d46da65d45c247 4dbcfba7e3c39a43.pdf

Ospina, W. (2013). Pa' que se acabe la vaina. Editorial Planeta Colombiana.

Pont, V. y Arango, M. (2017). Estado del arte sobre la corrupción en Colombia: Marco conceptual, diagnóstico y propuestas de política. Cuadernos de Fedesarrollo.

Rollón, M. y García, F. (2019). El control de la corrupción en América Latina: agenda política, judicialización e internacionalización de la lucha contra la corrupción. Documentos de trabajo (Fundación Carolina): Segunda época, (11).

Rúa, C. (2013). La legitimidad en el ejercicio del poder político en el Estado Social de Derecho. Una revisión desde el caso colombiano. Ius et Praxis, 19(2), 85-122. http://dx.doi.org/10.4067/S071800122013000200004

Rúa, C., Duarte, T. y Gómez, L. (2018). Escenarios para el sometimiento de excluidos de Justicia y Paz a la Jurisdicción Especial para la Paz: disquisición sobre sus ámbitos de aplicación. Opinión Jurídica, 17(35),117-145. https://revistas.udem.edu.co/index.php/opinion/article/view/2783

Sánchez, N. (2017). Corrupción y democracia. Universidad Autónoma de Barcelona. 
Sanguino Madariaga, A. (2008). Delitos contra la administración pública en la jurisprudencia. Librería Jurídica Sánchez.

Seligson, M. (2008). El Estado, la gobernabilidad y la legitimidad política en América Latina. En R. Mariani (Coord.), Democracia/Estado/Ciudadanía Hacia un Estado de y para la Democracia en América Latina (pp. 189-201). PNUD.

Seligson, M. y Smith, A. (2010). Cultura política de la democracia, 2010 Consolidación democrática en las Américas en tiempos difíciles: Informe sobre las Américas. Vanderbilt University.

Shah, A. y Schachter, M. (2004). Combating Corruption: Look before you leap. Finance E Development, 40-43.

Súarez, A. (2018). Colombia: de la corrupción común a la corrupción a gran escala. Revista Nova et Vetera, 4(37). https://www.urosario.edu.co/Revista-Nova-Et-Vetera/Omnia/Colombia-de-la-corrupcioncomun-a-la-corrupcion-a/

Transparencia Internacional. (2009). Guía de lenguaje claro sobre lucha contra la corrupción. Transparency International.

Transparency International. (2015). Transparency International statement: Recommendations for robust action against grand corruption.

Transparency International. (2020). Corruption Perception Index 2019. Transparency International.

Unodc (2004). The Global Programme against Corruption. Anti Corruption Toolkit; United Nations.

Uribe, S. (2012). Delitos contra la administración pública. Ediciones Unaula.

Vallejo, G. (2011). En busca de otro derecho penal. Opinión Jurídica, 10(19), 141-154. https://dialnet. unirioja.es/servlet/articulo?codigo $=3688503$

Vargas, O. (2017). Lo probable y lo demostrable. En Memorias XXXVIII Congreso de Derecho Procesal. Instituto Colombiano de Derecho Procesal. 